\title{
Surface Treatment with the Cold Roll Bonding Process for an Aluminum Alloy and Mild Steel
}

\author{
C. Tang, ${ }^{\mathrm{a}, 1}$ Z. Liu, ${ }^{\mathrm{a}}$ D. Zhou, ${ }^{\mathrm{b}}$ and S. Wu ${ }^{\mathrm{a}}$ \\ a School of Electro-Mechanical Engineering, Guangdong University of Technology, Guangzhou, \\ China \\ ${ }^{\mathrm{b}}$ Scientific Research Institute, Yinbang Metal Clad Material Co. Ltd, Wuxi, China \\ ${ }^{1}$ tangchl@gdut.edu.cn
}

The cold roll bonding process of a laminar metal composite consists of three steps, i.e., surface treatment, cold roll bonding, and heat treatment. The surface pretreatment is the precondition of obtaining a metal composite with a superior bonding level. The influence of the steel plate surface condition on the bonding strength is studied, based on the bonding mechanism of a laminar metal composite by cold roll bonding. Flap disc grinding is a better surface treatment than wire brushing for obtaining high bonding strength. Within a certain range, the larger the surface roughness, the higher the bonding strength. Comparing different grinding textures, viz longitudinal, transverse, and $45^{\circ}$ with respect to the rolling direction, it was revealed that the longitudinal surface texture was more advantageous for bonding.

Keywords: cold roll bonding, surface treatment, bonding strength, surface topography, surface texture, surface roughness.

Introduction. The air cooling system units of power stations are composed of finned tubes consisting of aluminum alloy fins welded together with the parent tube made of mild steel. However, brittle intermetallic compounds, which are formed at the weld interface during the process of brazing the aluminum alloy fins directly to the steel parent tube, deteriorate the joint quality. As a solution to this problem, a transition layer of aluminum is cladded over the steel surface by cold roll bonding: when this transition layer of aluminum is welded with the aluminum alloy fins instead of steel, a higher quality of the resulting joint is guaranteed.

The cold roll bonding process of $\mathrm{Al} / \mathrm{St}$ composites consisting of three steps (surface treatment, cold roll bonding and heat treatment), is bonding together the aluminum alloy and steel under high pressure by rolling at room temperature.

The bonding mechanism of cold roll bonding (CRB) is very complicated. Many experimental and theoretical studies imply that several bonding mechanisms are realized at the different stages of CRB process, such as: thin film formation [1-5], joint recrystallisation [6], dislocation, diffusion bonding, overcoming energy barriers, the three-stage mechanism, etc. The lack of persuasive experimental data and the complexiity of the CRB mechanisms give rise to numerous diversified yet insufficiently substantiated theories, which have the following common feature: the surface treatment is treated as the precondition to get high bonding strength of composites. In this study, the effect of surface treatment parameters, such as roughness, topography and texture directions of the metal surface, on the bonding strength of composite sheets are investigated.

At present, many studies had been performed on the factors affecting the bonding strength, such as reduction in thickness, rolling speed, annealing treatment, initial thickness, presence of particles between strips, etc. [6]. However, studies on the surface pretreatment are quite scarce. The surface condition of the metal to be bonded has a strong effect on the bonding strength. In the general case, metal surface is covered with a thin film composed of oxides, adsorbed ions, grease, moisture and dust particles. A surface treatment can provide 
not only the effective removal of the contamination layer covering the metal surface and uncover the bulk meta, but also favorable surface conditions for mechanical occlusion. It is well-known that currently available surface treatment methods have different effects on the surface state and bonding strength, while chemical treatment and mechanical grinding are considered to make the optimal combination of surface treatments, in case of cold roll bonding of metal composite sheets.

\section{Experimental Procedures.}

1.1. Materials. The materials under study are 08AL mild steel in the as-rolled state and 4A60 aluminum alloy in as-received state, chemical composition and mechanical properties of which are listed in Tables 1 and 2, respectively. As-received material blanks are cut along the original rolling direction to produce specimens of 4A60 aluminum alloy with dimensions $300 \times 75 \times 0.25 \mathrm{~mm}$ and $08 \mathrm{AL}$ mild steel with dimensions $300 \times 95 \times 3.75 \mathrm{~mm}$.

$\mathrm{T}$ a b 1 e 1

Chemical Composition of Aluminum Alloy and Mild Steel Sheet (wt.\%)

\begin{tabular}{|c|c|c|c|c|c|c|c|c|c||}
\hline \multirow{2}{*}{$08 \mathrm{AL}$} & $\mathrm{C}$ & $\mathrm{Mn}$ & $\mathrm{Si}$ & $\mathrm{S}$ & $\mathrm{Ni}$ & $\mathrm{Cr}$ & $\mathrm{Cu}$ & $\mathrm{Al}$ & $\mathrm{Fe}$ \\
\cline { 2 - 10 } & 0.03 & 0.212 & 0.014 & 0.004 & 0.007 & 0.015 & 0.026 & 0.004 & 99.68 \\
\hline \multirow{2}{*}{$4 \mathrm{~A} 60$} & $\mathrm{Si}$ & $\mathrm{Fe}$ & $\mathrm{Mn}$ & $\mathrm{Mg}$ & $\mathrm{Zn}$ & $\mathrm{Ti}$ & $\mathrm{Cu}$ & $\mathrm{Al}$ & Other \\
\cline { 2 - 10 } & 0.80 & 0.40 & 0.05 & 0.05 & 0.05 & 0.03 & 0.05 & 99.50 & $\leq 0.05$ \\
\hline
\end{tabular}

T a b 1 e 2

Mechanical Properties of Aluminum Alloy and Mild Steel Sheet

\begin{tabular}{|c|c|c|c|c||}
\hline Material & $\begin{array}{c}\text { Tensile strength } \\
(\mathrm{MPa})\end{array}$ & $\begin{array}{c}\text { Yield strength } \\
(\mathrm{MPa})\end{array}$ & $\begin{array}{c}\text { Elongation } \\
(\%)\end{array}$ & $\begin{array}{c}\text { Hardness } \\
(\mathrm{Hv})\end{array}$ \\
\hline $08 \mathrm{AL}$ & 470 & 460 & 11.0 & 130 \\
\hline $4 \mathrm{~A} 60$ & 85 & 45 & 19.4 & 37 \\
\hline
\end{tabular}

1.2. Surface Pretreatment. Many studies have shown that the best method of surface treatment is chemical degreasing before mechanical grinding. Therefore, in this study, chemical degreasing in 10\% hydrochloric acid bath and cleansing with acetone was used with the following mechanical grinding of specimen surfaces with an angle grinder $(850 \mathrm{~W}$ power, $72 \mathrm{~m} / \mathrm{s}$ rotating speed).

Comparision of the two bonded metals shows that the mild steel is much harder and less prone to plastic deformation than the aluminum alloy. Since the surface state of a mild steel is the key factor affecting the bonding strength, only the mild steel surface treatment parameters are investigated in this study.

1.3. Surface Roughness Measurement. The surface roughness of seven random areas on each specimen is measured with an OSL4000 confocal laser scanning microscope of Olympus according to ISO 25178 standard. The measurement area is $640 \times 640 \mu \mathrm{m}$; Gaussian filter is used to measure the arithmetic average deviation of surface roughness $(\mathrm{Sa})$. The maximum and minimum of the measurements are disregarded; the average value of the remaining five is taken as the surface roughness value.

1.4. Cold Roll Bonding. The CRB experiment is conducted via a reversible four-roller laboratory rolling mill with a loading capacity of 200 ton, a backup roll diameter of $400 \mathrm{~mm}$, and a work roll diameter of $170 \mathrm{~mm}$. The roll speed is $3 \mathrm{~m} / \mathrm{min}$ and reduction rate is $55 \%$. Specimens are stacked with rivets to avoid running deviations during the rolling process.

1.5. Tensile Shear Strength Test. According to the GB/T 6396-2008 standard, the bonding strength is evaluated via the tensile shear test, where the thickness of composite sheet is less than $10 \mathrm{~mm}$. The tensile shear test is carried out with an WDW-100 microcomputer-controlled servo-hydraulic universal tensile machine with the load cell of $50 \mathrm{~N}$ 
and displacement rate of $2 \mathrm{~mm} / \mathrm{min}$. However, in the conducted tensile shear tests, fracture often occurs in the aluminum layer, instead of the expected interface, because the aluminum layer is too thin. To increase its thickness, a $2 \mathrm{~mm}$-thick layer of 4343/3003 aluminum alloy was brazed over the aluminum layer. The tensile shear strength is calculated by the following formula:

$$
T=F_{\max } / A,
$$

where $T$ is the tensile shear strength, $F_{\max }$ is the maximum shear force $(\mathrm{N})$, and $A$ is the shear area $\left(\mathrm{mm}^{2}\right)$.

\section{Results and Discussion.}

2.1. Surface Topography of Different Grinding Methods. Surface topographies of a steel sheet subjected to flap disc grinding and wire brushing are shown in Figs. 1a and b, respectively. It is easy to see that the flap disc grinding produces very deep grooves and cracks at the steel surface, which makes it easier for the soft aluminum to squeeze into this surface and form a mechanical occlusion with steel. Moreover, the flap disc grinding produces a hard and brittle grind-hardened layer at the steel surface. According to the film theory, the governing mechanism in the cold roll bonding process is as follows: the brittle and hard layers break up along with the extension of the surfaces during rolling, while the internal fresh metal layers are extruded out through the cracks and exposed to contact with each other. Stable molecular bonds will be formed at the interface when the newly exposed surfaces of the two metals reach the interatomic distance under high enough pressure. On the other hand, the analysis of Fig. $1 \mathrm{~b}$ implies that wire brushing process fails to provide the complete removal of the original surface defects, while the resulting surface is less susceptable to bonding than the one produced via flap disc grinding. This is confirmed by the micrographs of the bonded steel surfaces after rolling presented in Figs. 2a and b for pretreatment by flap disc grinding and wire brushing, respectively. Cracks filled with the soft aluminum alloy at the steel surface pretreated by flap disc grinding are wider than those after wire brushing. This explains why the bonding strength of the laminar metal composite produced by flap disc grinding is higher than that pretreated by wire brushing, which is confirmed by the experimental results listed in Table 3.

T a b 1 e 3

\section{Tensile Shear Strength of Grinding Methods}

\begin{tabular}{|c|c|c||}
\hline Grinding methods & Tensile shear strength $\left(\mathrm{N} / \mathrm{mm}^{2}\right)$ & $S a(\mu \mathrm{m})$ \\
\hline Flap disc & 72 & 1.89 \\
\hline Wire brush & 64 & 1.78 \\
\hline
\end{tabular}
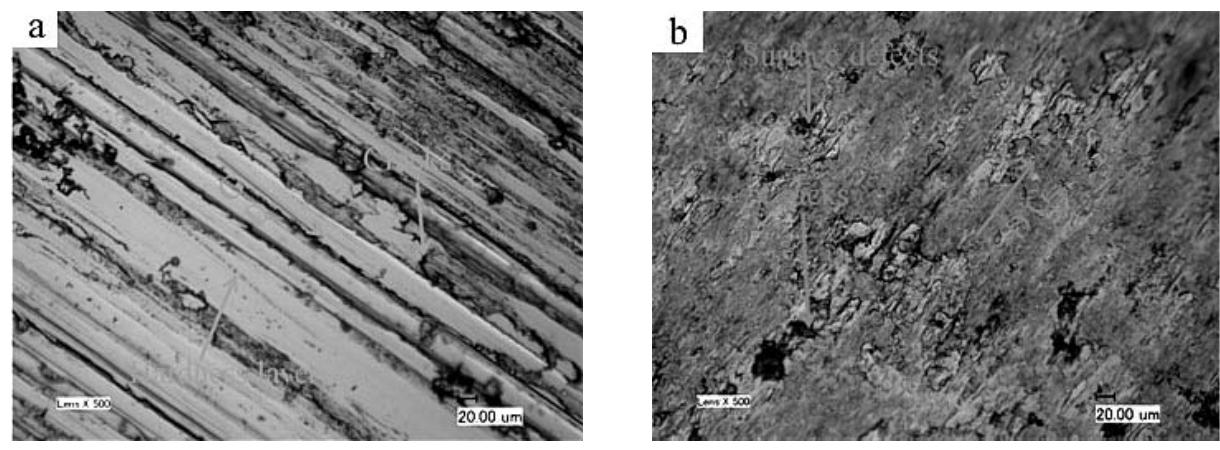

Fig. 1. Surface topography of a steel sheet before rolling $\times 1000$ : (a) flap disc grinding; (b) wire brushing. 

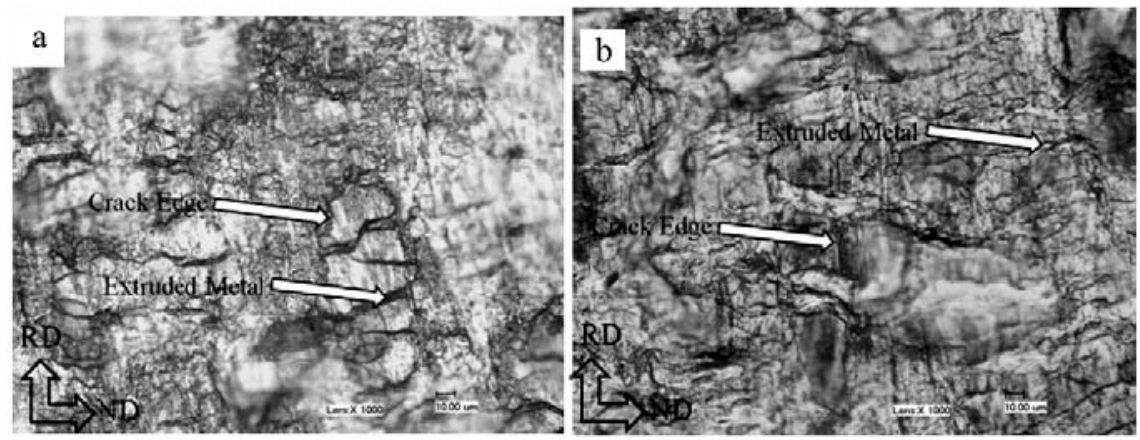

Fig. 2. Fracture surface of a steel sheet after rolling with $55 \%$ reduction $\times 1000$ : (a) flap disc grinding; (b) wire brushing.

The mechanical grinding can produce some peak-shaped structures protruding from the steel surface, which enhance the steel piercing into the adjoining aluminum alloy surface, as well as the mechanical occlusion contributing to the bonding process. 3D surface topographies depicted in Fig. 3 indicate that the above peaks and their amplitudes are significantly higher and their distribution along the surface is more uniform, in case of flap disc grinding, as compared to wire brushing. This trend is also corroborated by the SEM micrographs of the bonded steel surfaces, which are shown in Fig. 4.
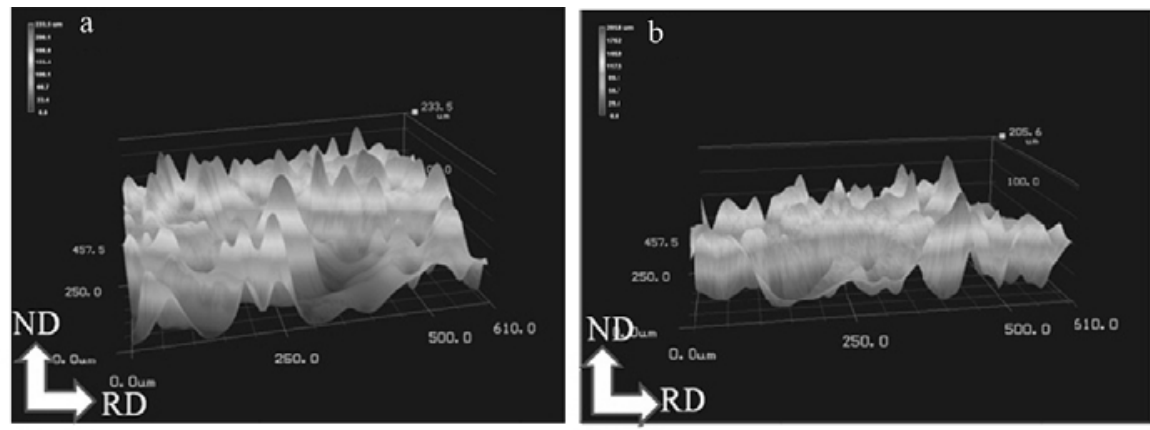

Fig. 3. 3D surface topography of a steel sheet $\times 1000$ : (a) flap disc grinding; (b) wire brushing.
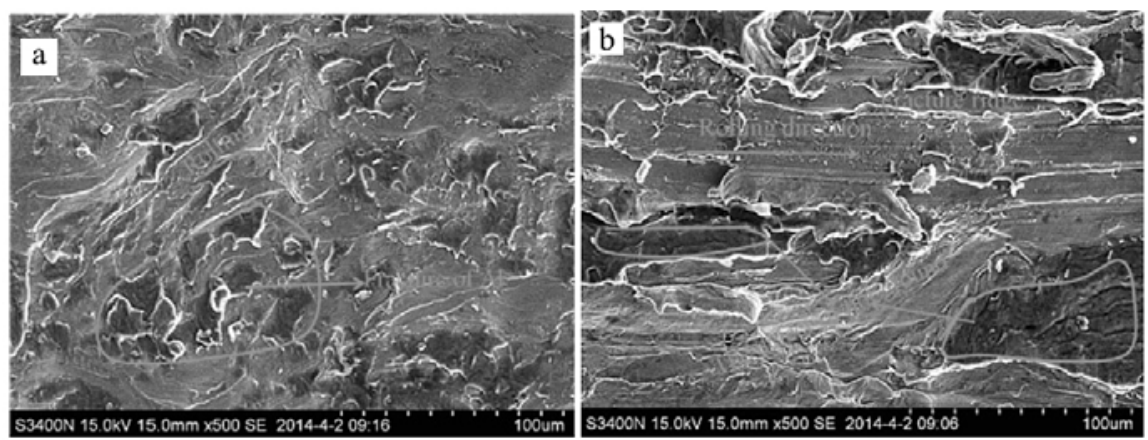

Fig. 4. SEM of the bonding surface of steel sheet $(R=55 \%) \times 500$ : (a) flap disc grinding; (b) wire brushing.

2.2. Effect of Surface Roughness on Bonding Strength. Five flap discs of different specifications (P40, P60, P80, P100, and P120) were chosen to grind the steel surface. The relationship between the bonding strength and roughness is shown in Fig. 5. The bonding strength is found to increase with the surface roughness within a certain roughness range. 


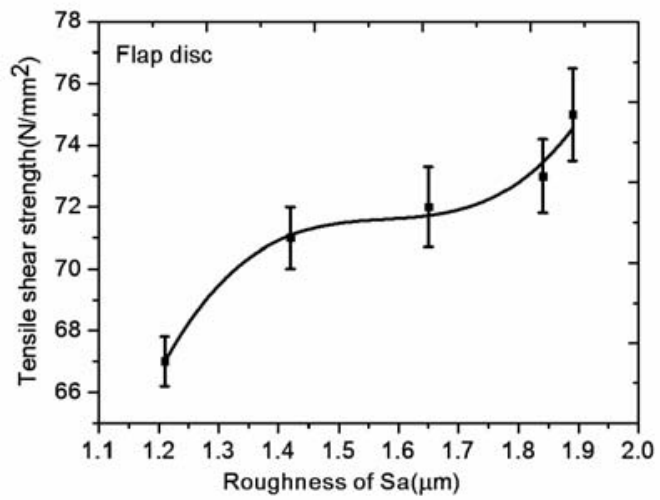

Fig. 5. Variation in average tensile strength vs surface roughness (flap disc grinding).

As shown in Fig. 6, long ridge-shaped grooves (about 32-45 $\mu \mathrm{m}$ deep) are produced at the steel surface by flap disc grinding, while more coarse flap discs produce deeper grooves at the ground steel surfaces.

In the CRB process, the contact area of the bonded metals is critical for the bonding strength. A rough surface can increase the contact area, while deep grooves can contribute to mechanical occlusion of the bonded metals, insofar as the localized shear deformation during the rolling process ensures the exposure of the bulk metals and their close contact. Thus, higher surface roughness, groove depth, and mechanical occlusion imply higher shear deformations, which, in turn, increase the bonding strength.
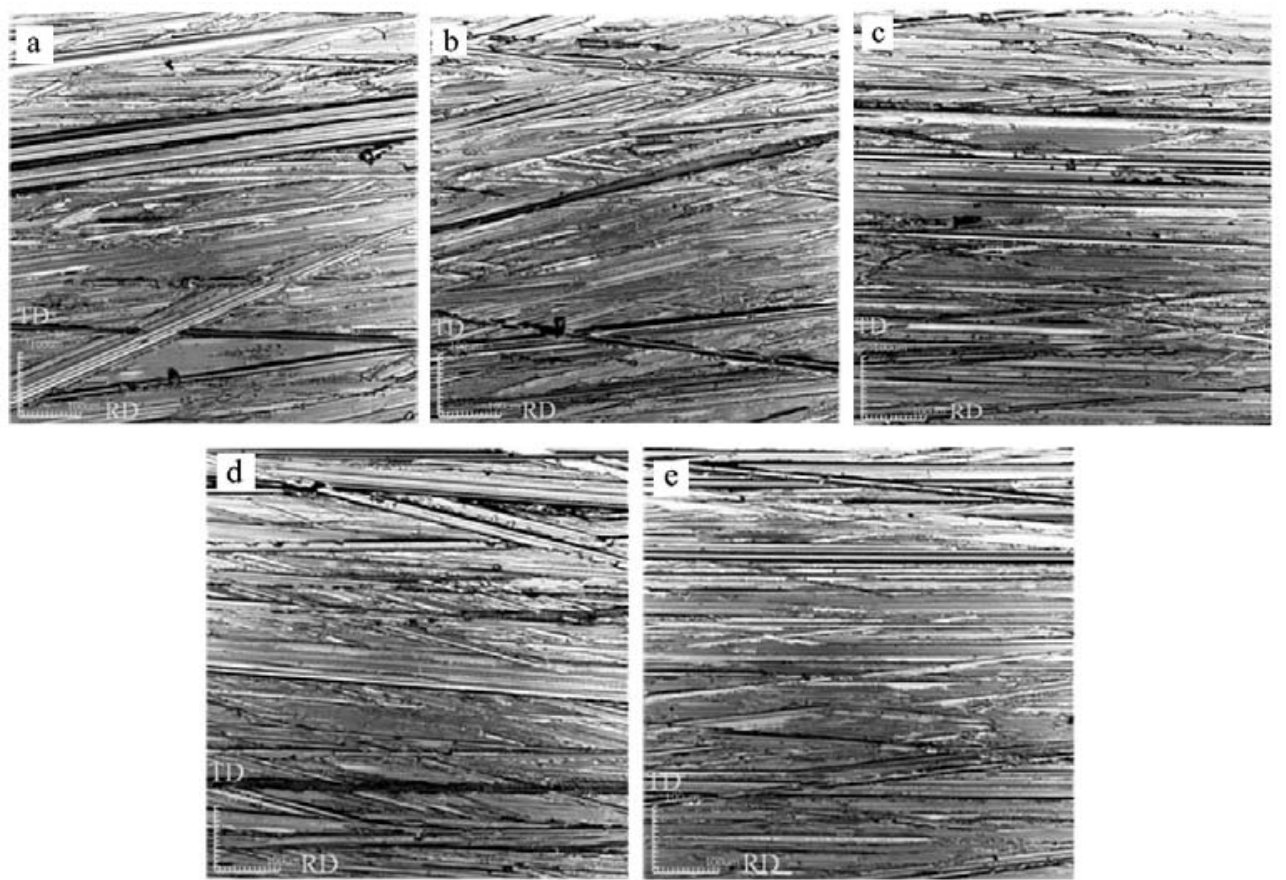

Fig. 6. Surface topography for flap disc grinding × 400: (a) P40; (b) P60; (c) P80; (d) P100; (e) P120.

2.3. Surface Texture Direction Effect on the Bonding Strength. The surface texture direction effect on the bonding strength is quite topical [6]. Therefore, three surface texture directions were investigated in this study: longitudinal, $45^{\circ}$-inclined, and transverse to the 
Surface Treatment with the Cold Roll Bonding Process ...

$\mathrm{T}$ a b 1 e 4

Tensile Shear Strength of Different Texture

\begin{tabular}{|c|c|c|}
\hline Texture direction & Tensile shear strength $\left(\mathrm{N} / \mathrm{mm}^{2}\right)$ & Roughness $S a(\mu \mathrm{m})$ \\
\hline Longitudinal & 74 & 1.17 \\
\hline $45^{\circ}$-inclined & 40 & 1.43 \\
\hline Transverse & 32 & 1.18 \\
\hline
\end{tabular}
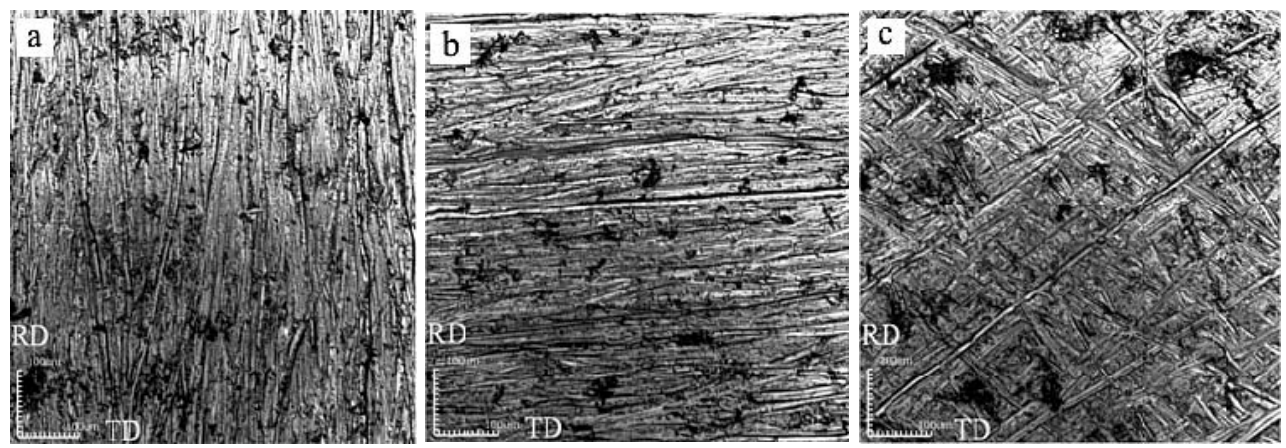

Fig. 7. Surface topography observed with a confocal laser scanning microscope $\times 400$ : (a) longitudinal texture; (b) transverse texture; (c) $45^{\circ}$-inclined texture.

rolling direction (Fig. 7). The experimental results on the tensile shear strength and surface roughness, which are listed in Table 4, indicate that respective surface roughness values are quite close, while the shear strength values can be arranged in the decreasing order as follows: longitudinal, $45^{\circ}$-inclined, and transverse texture directions.

According to the rule of the minimum roll bonding resistance, metal flow during the rolling process always occurs along the least-resistance direction. Since the resistance along the rolling direction is the least one, the texture direction of the metal surface coinciding with the rolling direction is the most lucrative for metal bonding, and, therefore, the longitudinal grinding surface texture is the most advantageous.

Conclusions. Based of the performed study of the effect of steel plate surface conditions on the bonding strength, the following conclusions can be drawn:

1. The comparative analysis of two surface treatment methods: flap disc grinding and wire brushing - was performed. The flap disc grinding process is found to be more advantageous for the subsequent cold roll bonding than wire brushing.

2. The relationship between the bonding strength and the surface roughness is investigated. It is shown that a certain surface roughness is necessary for cold roll bonding, and, within a certain range, a higher surface roughness results in a higher interface bonding strength.

3. Three surface texture directions were investigated: longitudinal, $45^{\circ}$-inclined, and transverse to the rolling direction. The tensile shear strength values can be arranged in the decreasing order as follows: longitudinal, $45^{\circ}$-inclined, and transverse texture direction. The longitudinal grinding surface texture is found to be the most advantageous for bonding.

Acknowledgments. The present study was financed by the National High-Tech Research and Development Program (2013AA031301) and Chinese National-International Scientific and Technological Cooperation Project (2013DFB50170). The authors would like to thank the University of Science \& Technology Beijing (USTB) for providing rolling mill and materials used in this work. 
1. D. Pan, K. Gao, and J. Yu, "Cold roll bonding of bimetallic sheets and strips," Mater. Sci. Technol., 5, 934-939 (1989).

2. H. Y. Wu, S. Lee, and J.Y. Wang, "Solid state bonding of iron-base alloy, steel-brass and aluminum alloy," J. Mater. Process. Technol., 75, 173-179 (1998).

3. V. Y. Mehr, M. R. Toroghinejad, and A. Rezaeian, "The effects of oxide film and annealing treatment on the bond strength of $\mathrm{Al}-\mathrm{Cu}$ strips in cold roll bonding process," Mater. Design, 53, 174-181 (2014).

4. L. R. Vaidyanath and D. R. Milner, "Significance of surface preparation in cold pressure welding of metals," Br. Weld. J., 7, 1-6 (1960).

5. H. A. Mohamed and J. Washburn, "Mechanism of solid state pressure welding," Weld. J., 54, 302-310 (1975).

6. R. Jamaati and M. R. Toroghinejad, "Cold roll bonding bond strengths: review," Mater. Sci. Technol., 27, 1101-1108 (2011). 